\title{
Polarized extremes and the confused centre: Campaign targeting of voters with correlation neglect
}

\author{
Gilat Levy, Inés Moreno de Barreda and Ronny Razin ${ }^{\text {I }}$
}

\begin{abstract}
We model the effect of competing political campaigns on the opinion of voters who exhibit correlation neglect, i.e., fail to understand that different campaigns might be correlated. We show that political campaigners can manipulate voters' beliefs even when voters understand the informativeness of each campaign separately. The optimal coordination of campaigns involves negative correlation of good news and sometimes full positive correlation of bad news. We show that competition in targeted campaigns has the effect of changing the opinions of different groups in different ways; competition increases polarisation among extreme voters but at the same time increases the variance and the quality of moderates' voting decisions.
\end{abstract}

\footnotetext{
${ }^{1}$ Levy: London school of Economics; Moreno de Barreda: University of Oxford; Razin: London school of Economics. We thank Clement Minaudier for excellent research assistance. This project has received funding from the European Union's Horizon 2020 research and innovation programme under grant agreement no 681579.
} 
In this day and age, we are constantly exposed to many information sources. As voters, we obtain news from different media sources, offline as well as online and through a myriad of social media platforms. This abundance of information might trigger simple heuristics that voters might use to aggregate multiple sources of information. A recent empirical literature documents, in different environments, how people exhibit "correlation neglect", a propensity to treat information sources as if they are (conditionally) independent. ${ }^{\square}$ Ortoleva and Snowberg (2015) documents how correlation neglect shapes political views, Eyster and Weizsäker (2011), Kallir and Sonsino (2009) and Enke and Zimmermann (2019) provides experimental evidence for such behaviours.

Voters that exhibit correlation neglect may be prone to manipulation by political campaigns. Intuitively, voters with correlation neglect respond more strongly to the repetition of similar pieces of information as they believe they are independently generated. This implies that to manipulate voters, campaigners have an incentive to coordinate and correlate multiple information sources. ${ }^{\text {[] }}$

In this paper we examine the implication of competition between two political campaigns who take advantage of voters with correlation neglect. We analyse a model of targeted and coordinated campaigns to influence voters' opinions, and derive its implications to the polarisation and quality of voters' opinions and decisions. We consider the following simple model of how voters' opinions are manipulated. There is a binary state of the world, and the public can decide between two actions. There are two political campaign strategists/coordinators, each supporting a different action. Each strategist coordinates a campaign consisting of $n$ messages (i.e., designs the correlation structure of $n$ signals). When voters observe the campaign messages they are able to interpret each of these individually (that is, they understand their accuracy), but they are not aware that messages are potentially correlated. Instead, they treat each campaign message as independent to the others. The campaign coordinators can also target messages to specific voters; they can differentiate between extreme voters (the "bases") and moderate voters (swing voters). We assume that the bases are only responsive to the campaign from their own side whereas swing voters listen to messages from both campaigns, and so both strategists target them.

Our results show that political campaigns have different effects on different voting groups. In par-

${ }^{2}$ De Marzo et al. (2003), Golub and lackson (2012), Gagnon-Bartsch and Rabin (2015) and Denter et al. (2019) study how correlation neglect affects the diffusion of information in social networks. Glaeser and Sunstein (20)(2) and Levy and Razin (2015a;b) explore the implications for group decision making in political applications.

${ }^{3}$ Indeed, in the last two years, more and more instances of orchestrated attempts to feed information into social media while hiding the true sources are exposed. For example in the UK, the Guardian has recently exposed documents describing "A series of hugely influential Facebook advertising campaigns that appear to be separate grassroots movements for a no-deal Brexit [...] The mysterious groups, which have names such as Mainstream Network and Britain's Future, appear to be run independently by members of the public and give no hint that they are connected. But in reality they share an administrator who works for Crosby's CTF Partners and have spent as much as č $1 \mathrm{~m}$ promoting sophisticated targeted adverts aimed at heaping pressure on individual MPs to vote for a hard Brexit”. For more on this see, https://www.theguardian.com/politics/2019/apr/03/grassrootsfacebook-brexit-ads-secretly-run-by-staff-of-lynton-crosby-firm. 
ticular, we show that the opinions of extreme voters become more polarised in the presence of political campaigns, in a predictable way. In contrast, the opinions of moderate voters become more unpredictable. The source of the unpredictability of moderate voters' opinions is the strategic noise that arises due to competition by the strategists over these voters. The implication of this extra noise is that the quality of moderates' voting behaviour is decreasing in the number of campaign messages; correlation neglect implies that these voters become worse at choosing the correct candidate as the arsenal of messages each strategist use expands.

Specifically, consider first extreme voters. These are voters that are ideologically tied to one side, and hence are the "base" of political support for that side. Even if their vote is somewhat guaranteed, what is important for political strategists is to intensify their beliefs so as to mobilise their vote as well as to use their activism to mobilise moderate voters. Thus, political campaign coordinators are interested in moving the beliefs of the base further in their direction. We show that correlation neglect implies increased polarisation. If voters were rational (weighing correlations correctly), the right-wing base and the left-wing base would have the same posterior expectations, while with correlation neglect and coordinated campaigns, both bases' expected opinions move further in the direction of their initial bias.

We show that the optimal way of correlating campaign messages targeted to the bases is to negatively correlate messages when the state is in the strategist's favour and positively correlate unfavourable news when the state goes against her. The intuition for this result stems from both correlation neglect and Bayesian persuasion. Correlation neglect implies that the voter's posterior is monotone in the number of pieces of news pointing to a particular direction. The posterior, represented as a function of the number of outlets with favourable information, takes the familiar S-shape of the value of information function. The strategist prefers to strongly correlate bad news some of the times, in order to be able to provide mildly good news most of the times. ${ }^{\text {T. }}$ We show that the optimal solution for the campaign follows a similar concavification intuition as in Kamenica and Gentzkow (201I), so that the positive and negative correlation results above follow from the concavification of the different parts of the S-shaped value function. The key result here then is that persuasion with correlation neglect results in the expected posterior moving away from the prior in a different direction for each of the base groups, hence creating polarisation.

Next, consider swing voters. These voters are "up for grabs", and vote for the side that convinces

${ }^{4}$ Our optimal information structure shares some features with the optimal solution in Harbaugh et al. (2017) albeit through very different mechanisms. Harbaugh et al. (20T7) analyse news' distortions when the receiver is rational but uncertain over the accuracy of the news and news are drawn independently. They show that when the news is mostly good, shoring up relatively bad news is most persuasive as it makes the good news appear more consistent and hence credible. But when the news is bad, exaggerating good news is better as bad news then appear less consistent. 
them that theirs' is the right course of action. Were these voters rational, the competition, as we show, would lead to full information revelation. On the other hand, when voters have correlation neglect, and view each message as independent, they simply vote for the action which has more supportive messages. This creates a tug of war between the two campaigns. Whatever one campaign tries to do, the other tries to undo. As we show, this zero sum game leads to strategic noise which diminishes the quality of these voters' voting decisions. In particular, the probability that the voters take the wrong action increases in the number of outlets each campaign can manipulate.

There is a large literature on voter polarisation, presenting mixed evidence. ${ }^{\text {[ }}$ Our paper is related to the effect of behavioural biases on voters' polarisation, as well as the strategic response of political actors to it, as recently explored in Levy and Razin (2015b), Ashworth and Bueno de Mesquita (2014), and Lockwood and Rockey (2018). Our focus is on the effect of correlation neglect in the presence of strategic coordination of campaigns and its targeting to different groups of voters. The literature on correlation neglect has focused indeed on how polarisation can arise when individuals ignore correlation across information sources (see Glaeser and Sunstein (2009), Levy and Razin (2015a), De Marzo et al. $(2003)$ and Ortoleva and Snowberg (2015)) ${ }^{\text {G }}$ In that literature, there is no strategic manipulation of beliefs.

We also contribute to the Bayesian persuasion literature, as we analyse persuasion when receivers have correlation neglect, ${ }^{\mathbb{D}}$ both when there is a single sender and with competing senders. For rational receivers, Kamenica and Gentzkow (2011) characterise the optimal signal that a single sender will design. In contrast with the rational receiver model, we show that persuasion takes place even when the sender has a linear utility over the posteriors of the receiver. ${ }^{\mathbb{8}}$ Gentzkow and Kamenica (2017) show that competition among senders always increases the information the receiver gets, as do Boleslavsky and Cotton (2014). Due to the rationality of receivers, in these models, competition implies more information is aggregated. In our model indeed when voters are rational, competition for moderate voters leads to full information revelation. However, when voters have correlation neglect, information aggregation under competition is far from perfect, and indeed decreases with the size of the campaigns. Mullainathan and Shleifer (2005) study the effect of competition on bias and information aggregation when readers have

\footnotetext{
${ }^{5}$ See Fiorina et al. (2005), Abramowitz and Saunders (2016), Harbridge and Malhotra (2011), Abramowitz (2016) and Layman and Thomas (2002).

${ }^{6}$ Sobel (2014) shows that a similar result can also arise for rational players. Roux and Sobel (2015), study a special case of Sobel (2014) with monotone environments, and similarly derive results about polarisation in group decisions when individuals are rational.

${ }^{7}$ For more general results about persuasion with correlation neglect see Levy et al. (2018).

${ }^{8}$ Meyer (2017) analyses how the heterogeneity of receivers affects the optimal persuasion of a sender (see also Ariell and Babichenko (2019)). She shows that the sender benefits from receivers being homogenous (or in the context of our model from using signals with the same marginal distribution) when the signals are private. Our model can be interpreted as one with many receivers who share their private information among themselves (or alternatively, when signals are public).
} 
confirmation bias. ${ }^{\square}$ They show that whether competition aggregates information depends on whether readers have divergent beliefs.

\section{The Model}

In the model there are two strategists $S_{L}, S_{R}$ who try to manipulate voters' beliefs, the first towards believing that the state is "Left" and the second that it is "Right", and a continuum of voters. Voters face a binary election about a policy in $\{L, R\}$ and their preferences depend on the state of the world.

Our model of manipulation captures the inherent trade-off between manipulation and credibility. In particular, we assume the following model. The strategists perfectly observe the state of the world and each of them coordinates $n>2$ different campaign outlets in order to influence the voters. A campaign outlet is interpreted as a signal about the state of the world. This could correspond to a news outlet, or a research institution that is affiliated with the campaign and sends occasional news stories or publishes research results. Voters know these outlets separately and expect them to yield signals about the world with a particular precision. The strategists control, to some degree, the joint information structure of the campaign outlets under their reach. In particular, they can coordinate the publication of the signals of each of these outlets but are constrained in the sense that each outlet needs to satisfy the voter's expected precision for the outlet. In other words, the campaigner can coordinate the messages of the different outlets as long as these outlets do not compromise, on average, their own reputation for sending informative messages. This implies that whatever the correlation structure the campaigner uses, when voters look at each outlet separately, they observe a signal which on average has the precision they expect from that outlet.

The voters use the campaign outlets' messages to learn about the state but they have correlation neglect. In other words, they perfectly understand the informational content (precision) of each campaign message coming from each outlet in isolation, but treat messages from different campaign outlets as if they were independent. For example, in the context of the UK campaign for leaving the EU, voters received messages from a website/group called 'Vote Leave' and another from a website/group called 'BeLeave'. While they might have understood the informativeness of each message separately, they probably did not have a good understanding of the level of coordination between these two organisations. We proceed with a simple model to capture the above.

Information and correlation neglect: Consider an environment with two states of the world, $\omega \in$

${ }^{9}$ Balan et al. (2009) provided a similar comparison of market structures, albeit with a model in which the behaviour of readers is black boxed. 
$\{L, R\}$ and a uniform prior which is common knowledge. The strategists observe the state and have at their disposal $n$ binary signals (campaign outlets) with realisations in $\{l, r\}$.

We assume that the accuracy of each signal is exogenously given. Campaigns often rely on the reputation of other sources to convey information to the public. This could be a study by a think-tank that they refer to, an article in a well known newspaper that they link to, or a citation by a well-known individual. The desire to be credible therefore constrains campaigns to given levels of credibility. Our assumption therefore is that the strategists' power is mainly to coordinate existing and credible sources, in order to focus on the effects of the correlation of campaigns.

Specifically, for a signal $s$, we denote its accuracy by $p=\operatorname{Pr}(s=\omega \mid \omega)>0.5$. For simplicity we assume homogenous signals that are symmetric across states. In turn, this last assumption allows us to attribute any bias in campaigns' influence to correlation of messages by the campaign. We assume that the accuracy of signals is common knowledge. In that sense, voters are not fully naïve as they understand the process according to which individual campaigns are correlated with the state of the world. However, voters are also not fully sophisticated; they fail to recognise the interdependence among campaign messages.

Before presenting the rest of the model, we introduce a preliminary result with regard to the beliefs of the voters who have correlation neglect. Given that all individual signals have the same accuracy and voters treat them as independent, the number of campaign messages with realisation $r$ is a sufficient statistic for the voters' beliefs. We denote by $V(k)$ the belief of the voter that $\omega=R$ when she observes $k$ realisations $r$ out of $n$ signals with accuracy $p$. Given the receiver's correlation neglect we have:

$$
V(k)=\frac{p^{k}(1-p)^{n-k}}{p^{k}(1-p)^{n-k}+(1-p)^{k} p^{n-k}} .
$$

The following is a helpful characterisation of $V(k)$.

Lemma 1. The voters' posterior, $V(k)$, is symmetric around $k=n / 2$, increasing, and concave (convex) above (below) $k=n / 2$.

All the proofs are found in the Appendix.

Intuitively, $V$ represents the value of information for a voter with correlation neglect. When a majority of signals has value $r(l)$, adding another $r$ signal has decreasing (increasing) returns. Figure U represents the voters' posterior as a function of $k$, the number of signals with realisation $r$.

Preferences: Voters differ in their preference parameter $\alpha \in[-1,1]$. A voter with preference parameter $\alpha$, that believes that the state is $\omega=R$ with probability $\mu$, prefers policy $R$ whenever $\mu+\alpha>\frac{1}{2}$ and 


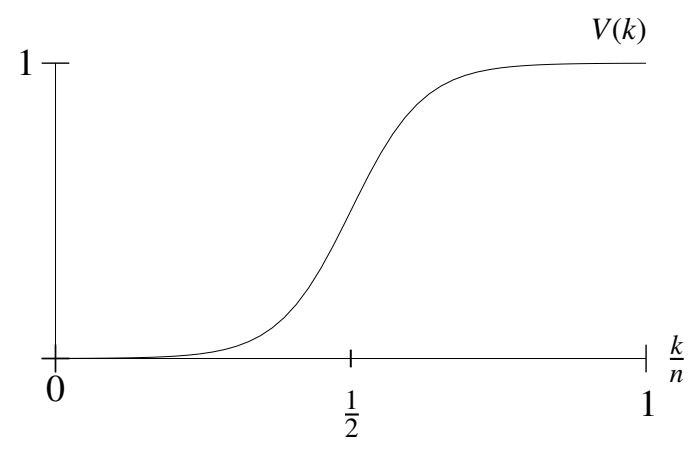

Figure 1: Voters' posterior belief that $\omega=R$ as a function of the proportion $\frac{k}{n}$ of signals with realisation $r$.

policy $L$ otherwise. In particular, the higher the parameter $\alpha$ the easier it is to convince a voter to choose policy $R$.

Given these preferences we divide the population into three groups. $B_{L}=\left\{\alpha \mid \alpha \in\left[-1,-\frac{1}{2}\right)\right\}$ represents the base of strategist $S_{L}$; these voters would always support policy $L$ independently of their beliefs. Analogously $B_{R}=\left\{\alpha \mid \alpha \in\left(\frac{1}{2}, 1\right]\right\}$ represents the base of $S_{R}$. Lastly, $S V=\left\{\alpha \mid \alpha \in\left[-\frac{1}{2}, \frac{1}{2}\right]\right\}$ is the set of swing voters that might change their vote depending on the information they receive. We assume that the preference parameter $\alpha$ is symmetrically distributed around 0 . In particular, the parameter value $\alpha_{m}=0$ corresponds to the median voter.

The objective of strategists is twofold. First, to win the election, each needs a majority of the swing voters to vote in their favour. Thus, the utility of $S_{R}\left(S_{L}\right)$ is increasing in the probability that the median voter votes for her, or in the probability that the posterior belief that $\omega=R$ of the median is greater (smaller) than half, given the messages the median voter observes. ${ }^{\text {[0 }}$

On the other hand, the strategists want to energise their base, i.e., they want to move the belief of their base toward their desired direction as much as possible. The argument for this latter goal is that a convinced base is easier to mobilise and could be instrumental in the campaign for swing voters. Thus, with regard to the base, we simply assume that the utility of $S_{R}\left(S_{L}\right)$ is linearly increasing in the posterior beliefs of the bases that $\omega=R(\omega=L)$.

Timing and strategies: We assume that the two strategists design their campaigns using the information design framework (see Kamenica and Gentzkow (2011)). For any state $\omega$, strategists simultaneously choose a correlation structure (a joint distribution) over $n$ campaign messages with accuracy $p$. We assume that strategists can target their own base as well as the swing voters. Our assumption that a strategist cannot target their opponent's base simplifies our analysis, and could be interpreted as the endogenous choice of websites and social media accessed by voters; base voters have strong preferences

\footnotetext{
${ }^{10}$ We use a tie breaking rule when the median voter's posterior belief is equal to $\frac{1}{2}$.

${ }^{11}$ The linearity of preferences is not crucial for our results. Any increasing utility which is not too concave will lead to similar results.
} 
and as in Mullainathan and Shleifer (2005), do not read the other side's propaganda. These bases can be then considered as the echo chambers in which only some types of messages get exposure and are propagated. An alternative interpretation is that, as Ali et al. (2019) argue, it could be very difficult for campaigners to reach voters with opposing viewpoints.

Formally, for any $\omega$, strategist $S_{R}\left(S_{L}\right)$ chooses two joint distributions, $\operatorname{Pr}_{B}(\cdot \mid \omega), \operatorname{Pr}_{S V}(\cdot \mid \omega)$ over the realisations of $\mathbf{s} \in\{r, l\}^{n}$, one targeted at base voters and the other at swing voters, such that each such joint distribution satisfies the marginal constraint given by the accuracy of the signals, i.e. for each $s_{i} \in\{r, l\}, \operatorname{Pr}\left(s_{i}=\omega \mid \omega\right)=p$.

We proceed to analyse the competition between strategists and its effects when voters have correlation neglect. We compare our results to the case in which voters are rational. We show that when strategists can coordinate their campaign and target specific groups of voters, correlation neglect implies that the bases are more polarised, and the swing voters are less informed or more confused than in the rational voters case. We start from the analysis of the optimal campaigns vis a vis the bases, and then we move to the case of the swing voters (targeting implies that we can analyse each separately).

\section{Intensifying the Bases}

We start our analysis by studying the optimal campaign of a strategist that is addressing her own base. As the problem faced by the two strategists is symmetric we focus on $S_{R}$ whose goal is to persuade her base that the state is $\omega=R$, independent of the realisation of the state. $S_{R}$ 's utility is linearly increasing in $V(k)$.

Note that given the linearity of preferences, the strategist never benefits from campaigning when facing rational voters. The reason is that for any information structure the law of iterated expectation should hold and hence the expected posterior of a fully Bayesian voter should equal the prior. This is summarised in the following remark.

Remark 1. When facing rational voters, the expected posterior of the base equals the prior. There is no effect of campaigning on the beliefs of the base.

Consider now the case of voters with correlation neglect. In this case and given the way the voters update their information illustrated in Figure M, the strategist can use the convexity of the belief function in her favour.

\footnotetext{
${ }^{12}$ With non-linear preferences, a strategist might be able to persuade a rational voter. In those cases, correlation neglect introduces further convexities in the belief function making voters even more vulnerable to persuasion.
} 
The optimal solution is achieved by finding the concavification of $V(k)$ taking into account that the marginal probability of a signal having realisation $r$ is $p$ if the state is $\omega=R$ and $1-p$ if $\omega=L .^{\square]}$

In order to characterise the optimal solution, define $k^{*}>0$ as the point that satisfies $\frac{\partial V\left(k^{*}\right)}{\partial k}=\frac{V\left(k^{*}\right)-V(0)}{k^{*}}$, i.e., $k^{*}$ is the smallest positive point at which the concavification of $V$ joins $V$ itself. For simplicity assume that $n p$ and $k^{*}$ are integers. Suppose that the accuracy $p$ is relatively high so that $k^{*} \leq n p$. In this case, in state $\omega=R$, it is optimal to produce exactly $n p$ signals with realisation $r$. Producing more than $n p$ positive campaigns would need to be compensated by producing less than $n p$ positive campaigns some of the time in order for the marginal constraints to be satisfied. Since the belief function $V$ is concave at $n p$, there are diminishing benefits of introducing this risk. On the other hand, if the accuracy is relatively low, $k^{*}>n p$, the benefits of providing more than $n p$ good news some of the times more than compensates the diminishing loses of providing fully correlated negative news. When the realised state is $\omega=L$, in expectation $n(1-p)$ signals have realisation $r$. Since $n(1-p)$ is on the convex part of $V$, it is also optimal to mix between some good news some of the time and fully correlated negative news the rest of the time. This is illustrated in Figure $\square$ below.

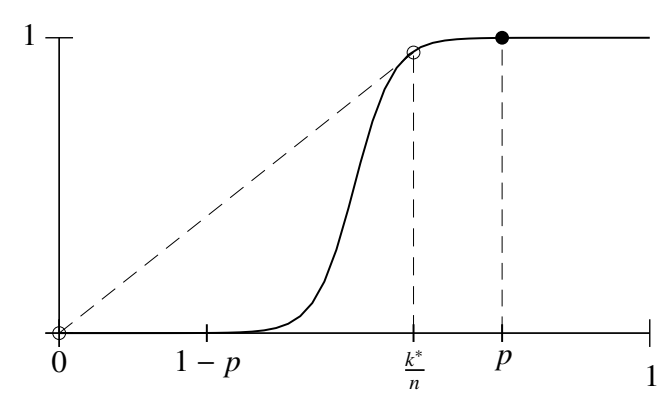

(a) Marginal $p=0.75$

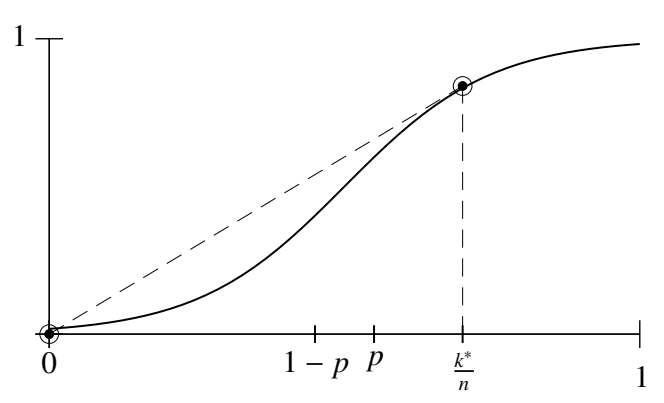

(b) Marginal $p=0.55$

Figure 2: Optimal joint distribution given the marginal constraint. The solid (hollow) dots represent the positive probability mass points when the state is $\mathrm{R}(\mathrm{L})$.

Proposition पा summarises the discussion.

Proposition 1. The optimal correlation structure for the strategist $S_{R}$ is given by:

1. If $p \geq \frac{k^{*}}{n}$,

- In state $\omega=R$, exactly np campaigns have realisation $r$.

- In state $\omega=L$, with probability $\frac{n(1-p)}{k^{*}}$, exactly $k^{*}$ campaigns have realisations $r$ and with probability $1-\frac{n(1-p)}{k^{*}}$, all campaigns have realisation $l$.

\footnotetext{
${ }^{13}$ Intuitively, the results are related to those in Kamenica and Gentzkow (20II). Although in the current analysis the utility of the sender is linear in the receiver's posterior, correlation neglect implies that this posterior has the usual S-shape of the value of information function. In addition, the Bayesian plausibility constraint in Kamenica and Gentzkow (20)II) is here replaced by the marginal constraints.
} 
2. If $p<\frac{k^{*}}{n}$,

- In state $\omega=R$, with probability $\frac{n p}{k^{*}}$, exactly $k^{*}$ campaigns have realisations $r$ and with probability $1-\frac{n p}{k^{*}}$, all campaigns have realisation $l$.

- In state $\omega=L$, with probability $\frac{n(1-p)}{k^{*}}$, exactly $k^{*}$ campaigns have realisations $r$ and with probability $1-\frac{n(1-p)}{k^{*}}$, all campaigns have a realisation $l$.

In either case the voters' expected belief is higher than the prior: $E[V(k)]>\frac{1}{2}$.

Overall, we expect good news to be negatively correlated (so that whenever some signal realisations are $r$, some are $l$ ), while bad news are sometimes fully positively correlated (so that all signal realisations are $l$ ).

Note that in contrast to the case of rational voters, when facing voters with correlation neglect the strategist manages to change the expectation of voters' posteriors away from the prior. In other words, the campaign is successful in intensifying the beliefs of her base.

Remark 2. A strategist facing a base with correlation neglect manages to intensify their beliefs.

The optimal information structure of $S_{L}$ is just a mirror image of what $S_{R}$ does. In other words, $S_{L}$ negatively correlates signals with realisation $l$ and sometimes fully correlate signals with realisation $r$, and by doing so $S_{L}$ manages to move the beliefs of $B_{L}$ towards $\omega=L$. The targeting of the bases therefore results in the polarisation of beliefs. Figure 31 represents the polarisation of beliefs of the two bases contingent on the realisation of the state for the case in which $p>\frac{k^{*}}{n}$.

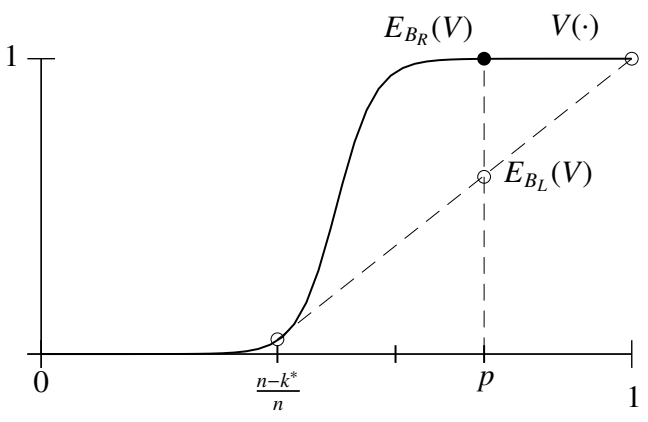

(a) $\omega=R$

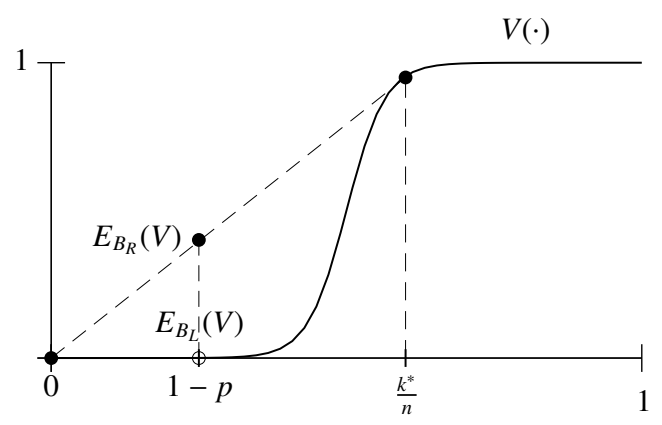

(b) $\omega=L$

Figure 3: Targeted campaigns for $p=0.75$ : Solid dots correspond to the campaign by $S_{R}$ and hollow dots to corresponds to the campaign by $S_{L}$.

As can be seen from the figure the two bases end up with very different expected beliefs, unlike the case of rational voters in which both bases' expected posterior equal the prior. Thus when voters are rational, there is no polarisation of the bases, while when they have correlation neglect, polarisation 
arises. Specifically, we measure the polarisation between the base as the distance between the beliefs given the state of the world. For $p>\frac{k^{*}}{n}$, this is given by:

$$
E_{B_{R}}[V]-E_{B_{L}}[V]=V(n p)-\left[\left(1-\frac{n(1-p)}{k^{*}}\right) V(n)+\frac{n(1-p)}{k^{*}} V\left(n-k^{*}\right)\right]>0
$$

Remark 3. When the bases have correlation neglect, polarisation arises.

In the limit, as the number of campaigns increases, the polarisation increases to $2(1-p) .^{\text {प] }}$ In particular, more precise campaigns ( $p$ close to 1 ) bounds how much strategists are able to manipulate the beliefs of the voters, whereas very uninformative campaigns ( $p$ close to $\frac{1}{2}$ ) are the ones that lead to higher polarisation of the voters.

\section{Persuading Swing Voters}

We now analyse the case in which the strategists target swing voters in order to persuade them to vote in their favour. As a benchmark, and in order to see the effect of competition, we first analyse the optimal information structure of $S_{R}$ when she is the sole strategist. ${ }^{[5]}$ We then introduced the two strategists and compute the equilibrium when they compete to attract swing voters.

\subsection{A Single Strategist}

Given the voters' preferences specified above, the voters' choice is weakly monotonic in their preference parameter $\alpha$; the higher the $\alpha$ the lower the number of signals needed in favour of $\omega=R$ in order to support policy $R$. The voting behaviour of a voter with preference parameter $\alpha$ is represented in Figure 因.

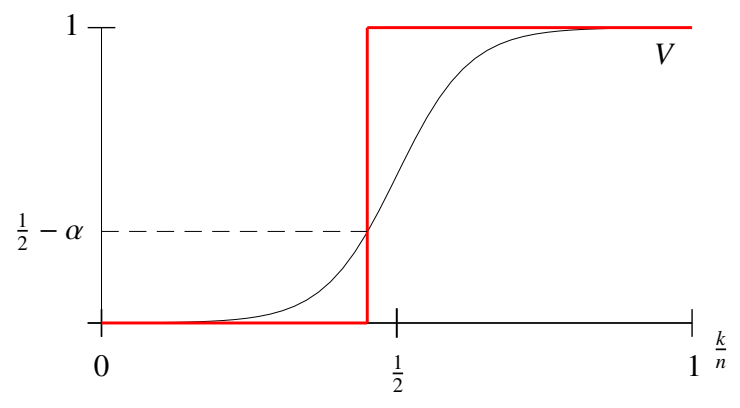

Figure 4: Choice of a voter with preference parameter $\alpha$.

\footnotetext{
${ }^{14}$ As the number of campaigns increases, $V(\cdot)$ converges to a step function with value 0 for $k<\frac{n}{2}$ and 1 for $k>\frac{n}{2}$. Therefore, as $n$ increases, $\frac{k^{*}}{n}$ converges to $\frac{1}{2}$. Hence, for any $p>\frac{1}{2}$ there exists a sufficiently large $n$ such that $p>\frac{k^{*}}{n}$.

${ }^{15}$ The optimal information structure for $S_{L}$ if she was the unique player is a mirror image of the one of $S_{R}$.
} 
To keep the model symmetric we assume, as specified before, that the preference parameter of the median voter is $\alpha_{m}=0$. In that case the strategist $S_{R}$ wants to maximise the probability of inducing more than $\frac{n}{2}$ positive signals, and hence convince the median voter. Using the same concavification techniques as in Proposition W we compute the optimal strategy for $S_{R}$.

Proposition 2. The optimal correlation structure for the strategist $S_{R}$ is given by:

- In state $\omega=R$, exactly np campaigns have realisation $r$.

- In state $\omega=L$, with probability $\frac{2 n(1-p)}{n+2}$, exactly $\frac{n}{2}+1$ campaigns have realisations $r$ and with probability $1-\frac{2 n(1-p)}{n+2}$, all campaigns have realisation $l$.

The probability of choosing policy $R$ is $\frac{1}{2}+\frac{n(1-p)}{n+2}>\frac{1}{2}$.

A single strategist is able to persuade swing voters to vote for her preferred action more than half of the times. ${ }^{\text {प6 }}$

We now move to our main result in this section, regarding competition between two strategists.

\subsection{Competition Between Two Strategists}

Consider now the case in which the two strategists compete for swing voters; to maintain our notation so that we can use $V(k)$, assume that each of them controls $\frac{n}{2}$ campaigns while the swing voters observe all these $n$ campaigns. The competition between strategists leads to a zero sum game, each strategist's payoff being 1 minus the other strategist's payoff.

If voters were rational, the unique equilibrium outcome of the competition game is such that the state is fully revealed. Each candidate uses a strategy in which the state is fully revealed by committing to information structures with disjoint support in each state, which rational voters can identify. ${ }^{\square}$ When $S_{L}$ fully reveals state $L$, the best $S_{R}$ can do is reveal state $R$. As a result, full revelation is indeed an equilibrium.

Remark 4. When swing voters are rational, competition between strategists perfectly reveals the state. ${ }^{\square 8}$

\footnotetext{
${ }^{16}$ Note that given the binary action choice, a strategist would be able to persuade rational swing voters as well, as in Kamenica and Gentzkow (201I). In fact, an alternative information structure, that replicates the one in Proposition $\square$ in state $\omega=L$ and mixes between $\frac{n}{2}+1$ signals $r$ with probability $\frac{2 n(1-p)}{n-2}$ and all signals $r$ with the remaining probability in state $\omega=R$, persuades a rational median voter to vote for $R$ with probability $\frac{1}{2}+\frac{n(1-p)}{n+2}$ and is still optimal when facing voters with correlation neglect.

${ }^{17}$ Remember that the strategists commit in advance to their strategies, so the voters never observe something unexpected.

${ }^{18}$ An example of such an equilibrium is one in which each strategist produces exactly $\frac{n}{2} p$ signals $r$ in state $\omega=R$ and $\frac{n}{2}(1-p)$ signals $r$ in state $\omega=L$. Since the voters observe two completely distinct behaviour in state $\omega=R$ than in state $\omega=L$, the state is fully revealed.
} 
Suppose now that voters have correlation neglect, i.e., the median voter votes for $R$ whenever more than half of the signals are $r$. As a result $S_{R}$ tries to increase the number $k$ of signals with realisation $r$ while $S_{L}$ tries to reduce it. This tug of war leads to an equilibrium in which the distribution over realisations contains large levels of noise. This is because if one strategist chooses an interior number of signals $r$ with high probability, the other strategist can always counteract this and pull the median voter's decision in her direction. It is precisely because of correlation neglect that this happens; correlation neglect implies that the voter only looks at the number $k$ of realisations $r$. Hence a simple zero sum game over the level of $k$ arises.

In order to complete the model we need to specify the behaviour of the median voter when she observes the same number of outlets with realisation $r$ and $l$. The nature of the marginal constraints, which depend on the realisation of the state, creates problems with existence of equilibrium, when the tie-breaking rule is state-independent. To guarantee existence of equilibrium for all $n$ and $p$, we assume then that when the voter is indifferent she takes the action which is in line with the state of the world. This assumption can be micro-founded by allowing the voter to access, prior to voting, a costly signal about the state of the world.

The following proposition describes the unique mixed equilibrium of this zero sum game. Intuitively, they uniformly mix over all possible realisations of $k$ besides the extremes $k \in\left\{0, \frac{n}{2}\right\}$ to which they place higher weight in the corresponding state in order to satisfy the marginal constraint.

Proposition 3. The unique equilibrium of the competition game consists of both strategists using the same strategy, given by:

- In state $\omega=R$, they send $k \in\left\{0, \ldots, \frac{n}{2}-1\right\}$ signals with realisation $r$ with probability $\frac{4(1-p)}{n+2}$ and they send $k=\frac{n}{2}$ signals with realisation $r$ with probability $1-\frac{n}{2} \frac{4(1-p)}{n+2}$.

- In state $\omega=L$, they send $k \in\left\{1, \ldots, \frac{n}{2}\right\}$ signals with realisation $r$ with probability $\frac{n}{2} \frac{4(1-p)}{n+2}$ and they send $k=0$ signals with realisation $r$ with probability $1-\frac{n}{2} \frac{4(1-p)}{n+2}$.

The probability that the electorate chooses the right option is $1-\frac{2 n(1-p)^{2}}{(n+2)}<1$.

Note that the equilibrium strategy in state $\omega=L$ coincides with the strategy when $\omega=R$ except for swapping the probabilities of sending all signals with realisation $r$ and all signals with realisations $l$. In particular, the symmetry of the strategies implies that the expected posterior of the voters (unconditional on the state) equals the prior. In other words, the competition among strategists eliminates the overall

\footnotetext{
${ }^{19}$ In Appendix B we provide the details of such micro-foundation. Intuitively, if voters have access to a costly signal that they can acquire before voting, they would only do so when the information she gleans from the campaigns is inconclusive, i.e., when an equal number of campaign messages push in two different directions.
} 
bias in the campaigns. However, in contrast with the case of rational voters, competition does not reveal the state and the presence of correlation neglect leads to confused swing voters. The tug of war between the strategists induces a high degree of mixing, and as a result, less information compared to the case of rational voters.

Remark 5. In the presence of correlation neglect, competition among strategists leads to confused swing voters.

Specifically, the probability that the median voter chooses the wrong action is $\frac{2 n(1-p)^{2}}{(n+2)}$. This increases in $n$, the number of overall messages that she is exposed to, and naturally decreases in $p$, the accuracy of each signal.

\section{Conclusion}

In this paper we analyse the competition between strategic political campaigns when voters have "correlation neglect". We show that the ability of campaigns to both target voters as well as coordinate political messages has different implications for extreme voters (over which there is little competition) to moderate ones (over which there is fierce competition). Our model shows that increased competition to manipulate voters introduces more noise. The result is that while extreme voters' opinions get more polarised with each other, moderates' opinions exhibit more state-independent variance. These results have both normative and positive implications. As the technology of voter manipulation increases (more messages, better ability to target voters) we would expect less information aggregation by campaigns and the correlation between the opinions of extreme and moderates should go down. The analysis in this paper can provide a foundation to the empirical study of how co-movements in public opinion of different voter groups relates to the exposure of such groups to different information sources. 


\section{A Appendix}

\section{A.1 Appendix A: Proofs}

\section{A.1.1 Proof of Lemma 11}

The symmetry around $k=\frac{n}{2}$ follows from $V(k)+V(n-k)=1$ for all $k$.

Taking the first and second derivative of $V(\cdot)$ with respect to $k$ we have:

$$
\begin{aligned}
& \frac{\partial V(k)}{\partial k}=2 \ln \left(\frac{p}{1-p}\right) \frac{p^{n}(1-p)^{n}}{\left[p^{k}(1-p)^{n-k}+(1-p)^{k} p^{n-k}\right]^{2}} \\
& \frac{\partial^{2} V(k)}{\partial k^{2}}=-4\left(\ln \left(\frac{p}{1-p}\right)\right)^{2} \frac{p^{n}(1-p)^{n}}{\left[p^{k}(1-p)^{n-k}+(1-p)^{k} p^{n-k}\right]^{3}}\left(p^{k}(1-p)^{n-k}-(1-p)^{k} p^{n-k}\right)
\end{aligned}
$$

Hence $V(k)$ is concave (convex) if and only if $p^{k}(1-p)^{n-k}-(1-p)^{k} p^{n-k}>0$ which is equivalent to $k>\frac{n}{2}$.

\section{A.1.2 Proof of Proposition 10}

Preliminary step: Denote by $k$ the number of signals with realisation $r . k$ is a sufficient statistic for the voters' expectation about the state of the world. Denote by $Q_{k}^{\omega}$ the total probability in state $\omega$ of having exactly $k$ signals with realisation $r$. We restrict attention to anonymous distributions, i.e., distributions that allocate the same probability to any vector of signal realisations with the same sum $\sum_{i=1}^{n} s_{i}=k$. This restriction is without loss of generality as anonymous distributions are optimal among all, including non-anonymous ones. Denote by $q_{k}^{\omega}$ the probability assigned by the sender to some specific $k$ signals having realisation $r$ and the others having realisation $l$, in state $\omega$. Then, $Q_{k}^{\omega}=\left(\begin{array}{l}n \\ k\end{array}\right) q_{k}^{\omega}$, and the marginal constraint can be written as:

$$
p^{\omega}=\operatorname{Pr}\left(s_{i}=r \mid \omega\right)=\sum_{k=1}^{n} \operatorname{Pr}\left(\sum_{j} s_{j}=k \mid s_{i}=r, \omega\right)=\sum_{k=1}^{n}\left(\begin{array}{l}
n-1 \\
k-1
\end{array}\right) q_{k}^{\omega}=\sum_{k=1}^{n} \frac{k}{n}\left(\begin{array}{l}
n \\
k
\end{array}\right) q_{k}^{\omega}=\sum_{k=1}^{n} \frac{k}{n} Q_{k}^{\omega}
$$

The strategist problem becomes finding the set of probabilities $\left\{Q_{k}^{\omega}\right\}_{k=0}^{n}$ with $Q_{k}^{\omega} \geq 0$ and $\sum_{k=0}^{n} Q_{k}^{\omega}=1$ to maximize $\sum_{k=0}^{n} Q_{k}^{\omega} V_{k}$ subject to the marginal constraint $\sum_{k=0}^{n} Q_{k}^{\omega} \frac{k}{n}=p^{\omega}$.

Lemma 2. The optimal distribution never allocates positive weights to more than two points. There exists $x, y \in\{0, \ldots, n\}$ such that if $k \notin\{x, y\} Q_{k}=0$.

Proof of Lemma 2]: Fix state $\omega$ and suppose that $Q_{x}, Q_{y}, Q_{z}>0$ for $x<y<z$ 미 Suppose we increase by $\varepsilon$ the weight on $y$, and hence reduce the weight on $x$ and $z$ together by $-\varepsilon$. To satisfy the marginal

\footnotetext{
${ }^{20}$ For simplicity of notation we ignore the reference to the state.
} 
constraint, we need $-x \beta+y \varepsilon-z(\varepsilon-\beta)=0$ which implies $\beta=\frac{\varepsilon(z-y)}{z-x}$. Note that we cannot have $\beta=0$ or $\beta=\varepsilon$. In terms of utility then, this is worth:

$$
\Delta_{x, y, z}=-V(x)\left(\frac{z-y}{z-x}\right)+V(y)-V(z)\left(1-\frac{z-y}{z-x}\right)
$$

If $V(y)>V(x)\left(\frac{z-y}{z-x}\right)+V(z)\left(1-\frac{z-y}{z-x}\right)\left(V\right.$ concave over these three points), $\Delta_{x, y, z}$ is positive and hence we can increase the objective function by moving weight from $x$ and $z$ towards $y$. If $V(y)<V(x)\left(\frac{z-y}{z-x}\right)+$ $V(z)\left(1-\frac{z-y}{z-x}\right)\left(V\right.$ convex over these three points), $\Delta_{x, y, z}$ is negative and we can increase the value of the objective function by moving weight from $y$ towards $x$ and $y$. If $V(y)=V(x)\left(\frac{z-y}{z-x}\right)+V(z)\left(1-\frac{z-y}{z-x}\right)$, by Lemma M, either there exists $v$ with $y<v<z$ such that $V$ is concave over $\{y, v, z\}$, and hence we can improve the objective function by decreasing $Q_{y}, Q_{z}$ and increasing $Q_{v}$, or there exists $v^{\prime}<x$ such that $V$ is convex over $\left\{v^{\prime}, x, y\right\}$ and we can improve the objective function by decreasing $Q_{x}$ and increasing $Q_{v^{\prime}}, Q_{y}$.

\section{Proof of Proposition 1}

Define $k^{*}$ such that $\frac{\partial V\left(k^{*}\right)}{\partial k}=\frac{V\left(k^{*}\right)-V(0)}{k^{*}}$. For simplicity we assume that $k^{*}$ and $n p$ are both integers. [1] Given Lemma $\square$ there are at most two positive Q's. Let's denote them by $Q_{m}, Q_{k}$, with $m \leq k$. By the marginal constraint we know that $m \leq n p \leq k$.

Suppose that $\frac{n}{2} \leq m \leq n p \leq k$, then by the concavity of $V(\cdot)$ above $\frac{n}{2}, \Delta_{m, n p, k}>0$ and we would benefit by moving all the weight to $n p$.

Suppose that $0<m<\frac{n}{2}<n p<k$, then $\Delta_{0, m, k}<0$ so we would benefit by moving weight to the extremes 0 and $k$.

Hence, the optimal information structure is either $Q_{n p}^{\omega}=1$ or $Q_{0}^{\omega}=1-\frac{n p^{\omega}}{k}, Q_{k}^{\omega}=\frac{n p^{\omega}}{k}$ for some $k>n p^{\omega}$. Which one is better depends on whether $n p^{\omega}<k^{*}$ or $n p^{\omega} \geq k^{*}$. Suppose that $n p^{\omega}<k^{*}$, then $\Delta_{0, n p^{\omega}, k^{*}}<0$ and putting weights on 0 and $k^{*}$ is better than all weight at $n p^{\omega}$. Moreover, $\Delta_{0, k^{*}, k}>0$ for all $k>k^{*}$ so the optimal information structure is $Q_{0}^{\omega}=1-\frac{n p^{\omega}}{k^{*}}, Q_{k}^{\omega}=\frac{n p^{\omega}}{k^{*}}$. Suppose that $n p^{\omega} \geq k^{*}$, then $\Delta_{0, n p^{\omega}, k}>0$ for all $k>n p^{\omega}$ and putting all weight on $n p^{\omega}$ is optimal.

Finally, the ex-ante expected belief is

$$
E[V(k)]=\frac{1}{2} E[V(k) \mid \omega=0]+\frac{1}{2} E[V(k) \mid \omega=1]>\frac{1}{2} V(n(1-p))+\frac{1}{2} V(n p)=\frac{1}{2} .
$$

\footnotetext{
${ }^{21}$ Otherwise we would replace $k^{*}$ by the highest integer such that $V(k)-V(0) \leq k(V(k)-V(k-1))$ and $n p$ by $k(p)$ and $k(p)-1$ $k(p)-1<n p<k(p)$.
} 


\section{A.1.3 Proof of Proposition 2}

The proof replicates the proof of Proposition $\square$ replacing $V(\cdot)$ by the step function

$$
a(k)=\left\{\begin{array}{ccc}
0 & \text { if } & V(k)<\frac{1}{2} \\
1 & \text { if } & V(k)>\frac{1}{2}
\end{array}\right.
$$

and where $k^{*}=\frac{n}{2}$.

\section{A.1.4 Proof of Proposition 3}

We provide the proof for $\omega=R$. The case $\omega=L$ is symmetric. We first show that the strategies proposed constitute an equilibrium. We start by showing that the strategies are feasible, that is, they satisfy the probability constraints (positive weights adding up to 1) and the marginal constraint. Using the notation of Proposition 1, the equilibrium strategies correspond to $Q_{k}=\frac{4(1-p)}{n+2} \equiv Q$ for $k \in\left\{0, . ., \frac{n}{2}-1\right\}$ and $Q_{\frac{n}{2}}=1-\frac{n}{2} \frac{4(1-p)}{n+2}=1-\frac{n}{2} Q$. It is easy to see that, $0<Q_{k}<1$ for all $0 \leq k \leq \frac{n}{2}$ and that $\sum_{k=0}^{\frac{n}{2}} Q_{k}=1$, so the strategies are indeed a probability distribution. The marginal constraint becomes:

$$
\sum_{k=0}^{\frac{n}{2}-1} Q \frac{k}{n / 2}+Q_{\frac{n}{2}}=p
$$

Replacing $Q$ and $Q_{\frac{n}{2}}$ by their values,

$$
\begin{aligned}
\frac{Q}{n / 2} \sum_{k=0}^{\frac{n}{2}-1} k+1-\frac{n}{2} Q & =\frac{Q\left(\frac{n}{2}-1\right)}{2}+1-\frac{n}{2} Q \\
& =1-\frac{Q\left(\frac{n}{2}+1\right)}{2}=p
\end{aligned}
$$

Finally, to see that this is an equilibrium note that the expected utility of $S_{R}$ when she sets $k_{R}$ signals with realisation $r$, and $S_{L}$ plays according with the strategy is:

$$
\begin{aligned}
V\left(k_{R}\right) & =\operatorname{Pr}\left(k_{L}+k_{R} \geq \frac{n}{2}\right) \\
& =\sum_{k_{L}=\frac{n}{2}-k_{R}}^{\frac{n}{2}} Q_{k_{L}} \\
& =1-\frac{4(1-p)}{n+2}\left(\frac{n}{2}-k_{R}\right)
\end{aligned}
$$


which is linear in $k_{R}$. To see that there are no profitable deviations from this strategy, consider another information structure for $S_{R},\left\{Q_{k_{R}}^{\prime}=Q_{k_{R}}+\Delta_{k_{R}}\right\}_{k_{R}=0}^{\frac{n}{2}}$. For the deviation to be feasible, it needs to satisfy the following two constraints:

$$
\sum \Delta_{k_{R}}=0, \quad \sum \frac{k_{R}}{n / 2} \Delta_{k_{R}}=0
$$

But then the change in the expected utility for $S_{R}$ given the deviation is:

$$
\begin{aligned}
\sum \Delta_{k_{R}} V\left(k_{R}\right) & =\sum \Delta_{k_{R}}-\sum \Delta_{k_{R}} \frac{4(1-p)}{n+2}\left(\frac{n}{2}-k_{R}\right) \\
& =\sum \Delta_{k_{R}}-\frac{n}{2} \frac{4(1-p)}{n+2} \sum \Delta_{k_{R}}+\frac{4(1-p)}{n+2} \sum k_{R} \Delta_{k_{R}} \\
& =0
\end{aligned}
$$

So there is no profitable deviation for $S_{R}$. An analogous computation shows that the expected utility of $S_{L}$ as a function of $k_{L}$ given what $S_{R}$ does and given the tie breaking rule, is also linear in $k_{L}$.

Finally, we show that this equilibrium is unique. Suppose that the strategies $\left\{Q_{k}^{S_{R}}\right\}_{k=0}^{n / 2}$ and $\left\{Q_{k}^{S_{L}}\right\}_{k=0}^{n / 2}$ constitute an equilibrium.

Claim 1: (i) If for $S_{R}, Q_{k}^{S_{R}}=0$ for some $k<\frac{n}{2}$ then for $S_{L}, Q_{\frac{n}{2}-(k+1)}^{S_{L}}=0$.

(ii) If for $S_{L}, Q_{k}^{S_{L}}=0$ for some $k<\frac{n}{2}$ then for $S_{R}, Q_{\frac{n}{2}-k}^{S_{R}}=0$.

Proof. Note that (ii) follows from (i) as they are mirror images. So we prove (i). Assume without loss that the state is $\omega=R$. Suppose that for some $k<\frac{n}{2}$, for $Q_{k}^{S_{R}}=0$. Note that by the tie breaking rule, whenever the sum of signals with realisation $r$ across both $S_{R}$ and $S_{L}$ are $\frac{n}{2}$ or higher $S_{R}$ 's utility is 1 . This implies that $S_{L}$ get the same level of utility from having $\frac{n}{2}-k$ signals with realisation $r$ as from having $\frac{n}{2}-(k+1)$. As the expected utility of $S_{L}$ is decreasing in the number of signals with realisation $r$, this implies that $\frac{n}{2}-(k+1)$ can never be part of an optimal solution for $S_{L}$; no concavication of his expected utility includes $\frac{n}{2}-(k+1)$ as a solution. Therefore, $Q_{\frac{n}{2}-(k+1)}^{S_{L}}=0$.

An implication of Claim 1 is that all equilibria are of the form whereby there exists a $k^{*}, p \frac{n}{2} \leq k^{*} \leq \frac{n}{2}$ such that the support of the strategy of $S_{R}$ is $\left\{0,1, \ldots, k^{*}\right\}$ and that of $S_{L}$ is $\left\{\frac{n}{2}-k^{*}, \frac{n}{2}-k^{*}+1, \ldots, \frac{n}{2}\right\}$.

ClaIm 2: Fix and equilibrium with $k^{*}$, for all $k<k^{*}, Q_{k}^{S_{R}}=\alpha, Q_{\frac{n}{2}-k}^{S_{L}}=\beta$

Proof. We provide a graphical proof. We know that the solution for $S_{R}$ is to concavify his expected utility $V^{S_{R}}(k)=\operatorname{Pr}\left(k_{L} \geq \frac{n}{2}-k\right)=\sum_{k_{L}=\frac{n}{2}-k}^{n / 2} Q_{k_{L}}^{S_{L}}$. The only way an optimal solution of this problem results in a support of $\left\{0,1, \ldots, k^{*}\right\}$ is if $V^{S_{R}}(\cdot)$ is linear on $\left\{0,1, \ldots, k^{*}\right\}$ which is what we need to prove. 
Consider $S_{L}$ and assume that $k^{*}<\frac{n}{2}$. Since $V^{S_{L}}\left(\frac{n}{2}-k^{*}\right)=-Q_{k^{*}}^{S_{R}}<0$ and $V^{S_{L}}(k)=-\sum_{k_{R}=\frac{n}{2}-k}^{k^{*}} Q_{k_{R}}^{S_{R}}$ for $k>\frac{n}{2}-k^{*}$ is linear, a convexification for $S_{L}$ implies an optimal solution with strictly positive weights on $\frac{n}{2}-k^{*}-1$ and $\frac{n}{2}$ only. This contradicts our premise of the equilibrium being characterised by $k^{*}$.

We conclude that the equilibrium has to have $k^{*}=\frac{n}{2}$ and by Claim 2 must take the form given in the proposition.

\section{A.2 Appendix B}

We conclude by motivating our tie breaking rule leading to the result of Proposition [3. We consider a modified game in which the electorate can, at a cost, choose an informative signal before they vote.

First we show that we can replicate the exact equilibrium we have introduced in the proposition by assuming that the signal the electorate have at their disposal is fully informative and its cost, $c$, is not too low and not too high. We then show more generally, that for other specifications of this model we get qualitatively similar behaviour in equilibrium. In particular, we provide another example in which the signal available is not fully informative, and moderate voters end up being even more confused than they are in the equilibrium in Proposition B.

Suppose first that the signal the electorate can acquire and is fully informative. Hence, if the median voter spends $c$, she gets a payoff $1-c$. In the absence of this extra signal, the expected utility of the median voter is $\max \{V(k), 1-V(k)\}$, which is lowest at $\frac{1}{2}$ when $k=\frac{n}{2}$, and second lowest at $\frac{p^{2}}{(1-p)^{2}+p^{2}}$ when $k=\frac{n}{2}+1$ or $k=\frac{n}{2}-1$.

Therefore, if $c$ is such that $\frac{1}{2}<1-c<\frac{p^{2}}{(1-p)^{2}+p^{2}}$ (or equivalently, $\frac{(1-p)^{2}}{(1-p)^{2}+p^{2}}<c<\frac{1}{2}$ ) then the equilibrium of this extended model corresponds to the equilibrium in Proposition [3].

More generally, the signal does not need to be perfectly revealing and the costs might be such that the electorate acquires the signal also when the number of campaign outlets in each direction is not exactly the same. We show in an example that the qualitative result of the equilibrium in Proposition [3] is maintained.

Consider $n=4$, and assume now that the signal the voter can acquire has accuracy $\beta$, i.e., the signals follows the correct state with probability $\beta$. Clearly, the median voter acquires this costly signal if and only if she would follow its recommendation. Let $\frac{(1-p)^{4}}{(1-p)^{4}+p^{4}}>\beta-c>\frac{p^{2}}{(1-p)^{2}+p^{2}}$. Then, the voter acquires and follows the signal unless in the political campaign she receives 4 realisations in favour of one of the options.

In this case we show that there is an equilibrium in which both strategists use the same strategy which mixes across all $k$ s. Suppose without loss of generality that the state is $R$. Denote by $\left\{Q_{0}, Q_{1}, Q_{2}\right\}$, where 
$Q_{k}$ is the probability of sending $k$ signals with realisation $r$, the strategy of $S_{L}$ in state $\omega=R$. Given the accuracy constraint and the fact that the probabilities have to add up to 1 , we can write $Q_{1}=2\left(1-p-Q_{0}\right)$ and $Q_{2}=Q_{0}+2 p-1$, with $0<Q_{0}<1-p$.

We now show that we can find $Q_{0}$ such that $U_{R}(2)-U_{R}(1)=U_{R}(1)-U_{R}(0)$. In other words, $S_{R}$ 's utility is linear in $k$, implying that $S_{R}$ is indifferent across all feasible strategies. In particular $S_{R}$ could use the same strategy as $S_{L}$, which would imply that $U_{L}(k) \equiv 1-U_{R}(k)$ is linear in $k$, and hence there is no profitable deviation for $S_{L}$ either.

For any $\frac{p^{2}}{(1-p)^{2}+p^{2}}+c<\beta<\frac{p^{4}}{(1-p)^{4}+p^{4}}+c$,

$$
\begin{aligned}
& U_{R}(2)=\left(Q_{0}+2\left(1-p-Q_{0}\right)\right) \beta+Q_{0}+2 p-1 \\
& U_{R}(1)=\beta \\
& U_{R}(0)=\left(1-Q_{0}\right) \beta
\end{aligned}
$$

Therefore, $U_{R}(2)-U_{R}(1)=U_{R}(1)-U_{R}(0)$ if and only if $Q_{0}=(1-\beta) \frac{2 p-1}{2 \beta-1}$. Which implies that both strategists choosing $\left\{Q_{0}=(1-\beta) \frac{2 p-1}{2 \beta-1}, Q_{1}=\frac{2(\beta-p)}{2 \beta-1}, Q_{2}=\frac{\beta(2 p-1)}{2 \beta-1}\right\}$ is an equilibrium. (Note that when $\frac{p^{2}}{(1-p)^{2}+p^{2}}+c<\beta<\frac{p^{4}}{(1-p)^{4}+p^{4}}+c$, the constraint $0<Q_{0}<1-p$ is satisfied.)

This equilibrium construction captures the spirit of the equilibrium we had before. First, the strategy of both strategists is the same. Second, the strategies are relatively uninformative. In particular, the probability that the correct decision is taken in state $R$ at the current equilibrium is:

$$
Q_{2}^{2}+\beta\left(1-Q_{0}^{2}-Q_{2}^{2}\right)=\beta+\beta(1-\beta) \frac{(2 p-1)^{2}}{2 \beta-1}
$$

This arises as when the state is $R$, the voter takes the correct state when both use $k=2$, the wrong state when both use $k=0$, and the correct state with probability $\beta$ in all remaining cases.

Setting $\beta=\frac{p^{2}}{(1-p)^{2}+p^{2}}$ (this is the lowest $\beta$ that satisfies the above constraints when $c=0$ ), we get that for $p<p^{\prime} \sim 0.7$ this probability is higher than $1-\frac{4(1-p)^{2}}{3}$, which was the probability of correct decision in the original equilibrium. 


\section{References}

Abramowitz, A. (2016). The Disappearing Center: Engaged Citizens, polarization, and American Democracy. New Haven, CT: Yale University Press.

Abramowitz, A. and K. Saunders (2016). Is polarization a myth? The Journal of Politics 70, 542-555.

Ali, M., P. Sapiezynski, A. Korolova, A. Mislove, and A. Rieke (2019). Ad Delivery Algorithms: The Hidden Arbiters of Political Messaging. Mimeo.

Arieli, I. and Y. Babichenko (2019). Private Bayesian Persuasion. Journal of Economic Theory 182, $185-217$.

Ashworth, S. and E. Bueno de Mesquita (2014). Is Voter Competence Good for Voters?: Information, Rationality, and Democratic Performance. American Political Science Review 108, 565-587.

Balan, D., P. DeGrava, and A. L. Wickelgren (2009). Ideological Persuasion in the Media. SSRN 637304.

Boleslavsky, R. and C. Cotton (2014). Limited Capacity in Project Selection: Competition through Evidence Production. Economic Theory, 1-37.

De Marzo, P., D. Vayanos, and J. Zwiebel (2003). Persuasion Bias, Social Influence and Unidimensional Opinions. The Quaterly Journal of Economics 118(3), 909-968.

Denter, P., M. Dumav, and G. Boris (2019). Social Connectivity, Media Bias and Correlation Neglect. Mimeo.

Enke, B. and F. Zimmermann (2019). Correlation Neglect in Belief Formation. Review of Economic Studies 86, 313-332.

Eyster, E. and G. Weizsäker (2011). Correlation Neglect in Financial Decision-Making. Mimeo.

Fiorina, M., S. Abrams, and J. Pope (2005). Culture War? Myth of a polarise America. Upper Saddle River, NJ: Pearson Education.

Gagnon-Bartsch, T. and M. Rabin (2015). Naïve Social Learning, Mislearning, and Unlearning. Mimeo.

Gentzkow, M. and E. Kamenica (2017). Competition in Persuasion. Review of Economic Studies 84(1), $300-322$.

Glaeser, E. L. and C. R. Sunstein (2009). Extremism and Solcial Learning. Journal of Legal Analysis $1(1)$. 
Golub, B. and M. O. Jackson (2012). How Homophily Affects the Speed of Learning and Best-Response Dynamics. The Quarterly Journal of Economics 127(3), 35-71.

Harbaugh, R., J. Maxwell, and K. Shue (2017). Consistent Good News and Inconsistent Bad News. Mimeo.

Harbridge, L. and N. Malhotra (2011). Electoral incentives and partisan conflict in congress: Evidence from survey experiments. American Journal of Political Science 55, 494-510.

Kallir, I. and D. Sonsino (2009). The Neglect of Correlation in Allocation Decisions. Southern Economic Journal 75(4), 1045-1066.

Kamenica, E. and M. Gentzkow (2011). Bayesian Persuasion. American Economic Review 101(6), $2590-2615$.

Layman, G. and C. Thomas (2002). Party polarisation and 'Conflict Extension' in the American Electorate. American Journal of Political Science 46(4), 786-802.

Levy, G., I. Moreno de Barreda, and R. Razin (2018). Persuasion with Correlation Neglect. Mimeo.

Levy, G. and R. Razin (2015a). Correlation Neglect, Voting Behaviour and Information Aggregation. American Economic Review 105(4), 1634-1645.

Levy, G. and R. Razin (2015b). Does Polarization of Opinions Lead to Polarization of Platforms? The Case of Correlation Neglect. Quarterly Journal of Political Science 10(3), 321-355.

Lockwood, B. and J. Rockey (2018). Negative voters: electoral competition with loss-aversion. Mimeo.

Meyer, M. (2017). Information Design: Insights from Orderings of Dependence and Heterogeneity. Mimeo.

Mullainathan, S. and A. Shleifer (2005). The Market for News. American Economic Review 95, 10311053.

Ortoleva, P. and E. Snowberg (2015). Overconfidence in Political Behavior. American Economic Review 105(2), 504-35.

Roux, N. and J. Sobel (2015). Group Polarization in a Model of Information Aggregation. American Economic Journal: Microeconomics 7(4), 202-232.

Sobel, J. (2014). On the Relationship between Group and Individual Decisions. Theoretical Economics 9, $163-185$. 